\title{
Use of Orthogonal Collocation Method for a Dynamic Model of the Flow in a Prismatic Open Channel: For Estimation Purposes
}

\author{
Asanthi Jinasena $^{1} \quad$ Glenn-Ole Kaasa $^{2}$ Roshan Sharma ${ }^{1}$ \\ ${ }^{1}$ Faculty of Technology, Natural Sciences and Maritime Sciences, University College of Southeast Norway, Norway \\ asanthi.jinasena@usn.no, roshan.sharma@usn.no \\ ${ }^{2}$ Kelda Drilling Controls A/S, Porsgrunn, Norway, gok akelda. no
}

\begin{abstract}
The modeling and simulation of free surface flows are complex and challenging. Especially, the open channel hydraulics are often modeled by the well-known and efficient Saint-Venant equations. The possibility of efficiently reducing these partial differential equations into ordinary differential equations with the use of orthogonal collocation method is studied with the goal of application in estimations. The collocation method showed the flexibility of choosing the boundary conditions with respect to the flow behavior. The results were comparable enough to the selected finite volume method. Further, a significant reduction in computational time in the collocation method is observed. Therefore, the collocation method shows a good possibility of using it for the real-time estimation of flow rate in an open channel.
\end{abstract}

Keywords: orthogonal collocation, open channel, prismatic, flow estimation, dynamic modeling

\section{Introduction}

The real-time estimation of flow rates in fluid flows with the use of mathematical models is a widely known practice in the industry, especially in oil drilling processes, hydro power industry and in agricultural industries. The simplicity and the robustness of the mathematical model are influential in estimation. However, the modeling and simulation of free surface flows are complex and challenging. Especially, the open channel hydraulics are often modeled by the well known and efficient shallow water equations, which are also known as the Saint-Venant Equations (SVEs). These are a set of nonlinear, hyperbolic Partial Differential Equations (PDEs). These equations are widely used throughout the history, yet the discretization remains tricky which makes it difficult to solve.

Although the classical methods such as finite difference and finite volume methods are of high precision, it needs numerous spatial discretization points to obtain a realistic solution and consumes a considerable amount of computational time. Hence, these numerical solvers could create complications in applications of online state and parameter estimation. On the contrary, the collocation method, which is a special case of the weighted residual method, could lead to simple solutions with less computational time. This method is commonly used in computational physics for solving PDEs and in chemical engineering for model reduction.

Therefore, the main aim of this work is to study the possibility of reducing the PDEs into Ordinary Differential Equations (ODEs) efficiently, with a future goal of an application in estimations. This paper describes the numerical approach which is taken to solve the 1-D shallow water equations in the reduced ODE form. Further, it includes the verification of the used numerical approach in comparison to the other well-known and accurate numerical schemes for selected case studies.

In this paper, the orthogonal collocation method is used for converting the PDEs into ODEs, and then the ODEs are solved using the Runge-Kutta fourth order numerical scheme (for the discretization in the time domain). The Lagrange interpolating polynomials are used for the approximation of the shallow water equations and the shifted Legendre polynomials are used for the selection of collocation points. For the case study, a prismatic channel with a trapezoidal cross-section along the length is selected as the open channel. Different numbers of collocation points were tested and the results are compared with the numerical simulation results obtained from a classical finite volume method. The finite volume method used in this study is a semi-discrete, second order and a central upwind scheme developed by Kurganov and Petrova (Kurganov and Petrova, 2007) for the spatial discretization and the Runge-Kutta fourth order numerical scheme for the temporal discretization.

\section{Mathematical Model}

There are a large number of versions of the SVEs, based on the physical natures those are assumed upon (Chalfen and Niemiec, 1986; Chaudhry, 2008). The SVEs are a set of hyperbolic, non-linear PDEs, and the used version of the SVEs in this study are derived with the assumptions listed below (Chaudhry, 2008; Litrico and Fromion, 2009).

- The pressure distribution is hydrostatic.

- The velocity of the flow is uniform over the cross section of the channel. 
- The channel is prismatic i.e. the cross sectional area perpendicular to the flow and the channel bed slope do not change with the direction of the flow.

- The channel bed slope is small i.e. the cosine of the angle it makes with the horizontal axis may be replaced by unity.

- The head losses in unsteady flow (due to the effect of boundary friction and turbulence) can be calculated through resistance laws analogous to those used for steady flow.

- No lateral inflow rates are considered.

The Equations for a 1D, unsteady, prismatic, open channel system, can be expressed as,

$$
\begin{aligned}
\frac{\partial A}{\partial t}+\frac{\partial Q}{\partial x} & =0, \\
\frac{\partial Q}{\partial t}+\frac{\partial\left(Q^{2} / A\right)}{\partial x}+A g\left(\frac{\partial z}{\partial x}+S_{f}-S_{b}\right) & =0
\end{aligned}
$$

where $A(x, h, t)$ is the wetted cross sectional area normal to the flow, $h(x, t)$ is the depth of flow, $Q(x, t)$ is the volumetric flow rate, $S_{f}(Q, x, h)$ is the friction slope, $z$ is the absolute fluid level, which changes with the geometry of the channel, $g$ is the gravitational acceleration, $t$ is the time and $x$ is the distance along the flow direction (Chow, 1959; Chaudhry, 2008). The channel bed slope $S_{b}(x)$ is calculated by $-\frac{\partial z}{\partial x}$, which is considered positive when sloping downwards. The friction slope $S_{f}$ is calculated from the Gauckler-Manning-Strickler formulae as shown in Equation 3 (Chow, 1959),

$$
S_{f}=\frac{Q|Q| n_{M}^{2}}{A^{2} R^{\frac{4}{3}}},
$$

where $n_{M}$ is the Manning friction coefficient $\left(\frac{1}{k_{s}}\right)$ and $R$ is the hydraulic radius given by $\frac{A}{P}$. Here, $k_{S}$ is the Strickler friction coefficient and $P$ is the wetted perimeter. The analytical solution for these equations exists only for the simplified cases (Chalfen and Niemiec, 1986; Chung and Kang, 2004; Bulatov, 2014), therefore, these are generally solved by numerical methods. Two different numerical methods are considered in this study, the orthogonal collocation method and the Kurganov and Petrova (KP) Scheme, which are described in the following sections 2.1 and 2.2.

\subsection{The Orthogonal Collocation Method}

The states $A$ and $Q$ in the SVEs can be approximated by the general polynomial interpolation, using the Lagrange interpolating polynomial (Isaacson and Keller, 1966). The Lagrange interpolating polynomial of $n^{\text {th }}$ order for a general function $f(x)$, at $n+1$ data points, is given by (Szegö, 1939),

$$
f_{n}(x)=\sum_{i=0}^{n} L_{i}(x) f\left(x_{i}\right)
$$

where,

$$
L_{i}(x)=\prod_{\substack{j=0 \\ j \neq i}}^{n} \frac{x-x_{j}}{x_{i}-x_{j}} .
$$

Here, $L_{i}(x)$ is a weighting function, which is considered as the basis function for the Lagrange function. Now, the approximated states can be defined as $A_{a}$ and $Q_{a}$, where,

$$
\begin{aligned}
& A_{a}(x, t)=\sum_{i=0}^{n} L_{i}(x) A_{i}(t), \quad \text { and } \\
& Q_{a}(x, t)=\sum_{i=0}^{n} L_{i}(x) Q_{i}(t) .
\end{aligned}
$$

Using these approximations in the SVEs, the Equations 1 and 2 can be re-written as follows,

$$
\begin{aligned}
\frac{\partial A_{a}}{\partial t}+\frac{\partial Q_{a}}{\partial x} & =R_{1} \\
\frac{\partial Q_{a}}{\partial t}+\frac{\partial\left(Q_{a}^{2} / A_{a}\right)}{\partial x}+A_{a} g\left(\frac{\partial z}{\partial x}+S_{f}-S_{b}\right) & =R_{2}
\end{aligned}
$$

where $R_{1}(x, \bar{A}, \bar{Q})$ and $R_{2}(x, \bar{A}, \bar{Q})$ are the residuals and $\bar{A}$ and $\bar{Q}$ are the vectors of the coordinates of $A_{a}$ and $Q_{a}$, respectively.

The spatial length $x$ is divided into $n-1$ inequidistant spaces for $n$ nodes, which are named as the collocation points. Two of these collocation points will be placed at the boundaries. When the residuals are closer to zero, the unknowns $(\bar{A}$ and $\bar{Q})$ can be computed for each collocation point $x_{i}^{c}$

$$
\begin{aligned}
& R_{1}\left(x_{i}^{c}, \bar{A}, \bar{Q}\right) \approx 0, \quad i=1,2, \ldots, n \\
& R_{2}\left(x_{i}^{c}, \bar{A}, \bar{Q}\right) \approx 0, \quad i=1,2, \ldots, n
\end{aligned}
$$

The corresponding collocation points $x_{i}^{c}$, can be found by choosing the points carefully. When the points are at the roots of any orthogonal polynomial such as the Legendre or Chebyshev polynomial, the approximation error can be minimized (Isaacson and Keller, 1966; Quarteroni and Valli, 2008). The Legendre polynomials are selected in this study. As the number of points are increased, these collocation points cluster towards the two endpoints of the selected total length. This prevents the formation of Runge's phenomenon, which occurs when the nodes are equispaced.

When the residuals are closer to zero, the Equations 8 and 9 can be re-written as follows,

$$
\begin{aligned}
\frac{\partial A_{a}}{\partial t}+\frac{\partial Q_{a}}{\partial x} & \approx 0, \\
\frac{\partial Q_{a}}{\partial t}+\frac{\partial\left(Q_{a}^{2} / A_{a}\right)}{\partial x}+A_{a} g\left(\frac{\partial z}{\partial x}+S_{f}-S_{b}\right) & \approx 0 .
\end{aligned}
$$

Further, the Equation 13 can be simplified as,

$$
\begin{aligned}
\frac{\partial Q_{a}}{\partial t} & +\frac{2 Q_{a}}{A_{a}} \frac{\partial Q_{a}}{\partial x}-\frac{Q_{a}^{2}}{A_{a}^{2}} \frac{\partial A_{a}}{\partial x} \\
& +A_{a} g\left(\frac{\partial z}{\partial x}+S_{f}-S_{b}\right) \quad \approx 0 .
\end{aligned}
$$


From the Equations 6 and 7, the derivatives are expressed as,

$$
\begin{aligned}
& \frac{\partial A_{a}}{\partial x}=\sum_{i=0}^{n} L_{i j}^{\prime} A_{i}, \quad \text { and } \\
& \frac{\partial Q_{a}}{\partial x}=\sum_{i=0}^{n} L_{i j}^{\prime} Q_{i},
\end{aligned}
$$

where

$$
L_{i j}^{\prime}\left(x_{i}\right)=\frac{\partial L_{i}(x)}{\partial x}
$$

The substitution of this expression in the Equations 12 and 14 will give two ODEs.

$$
\begin{aligned}
\frac{d A_{a}}{d t}+\sum_{i=0}^{n} L_{i j}^{\prime} Q_{i} & \approx 0, \\
\frac{d Q_{a}}{d t}+\frac{2 Q_{a}}{A_{a}} \sum_{i=0}^{n} L_{i j}^{\prime} Q_{i}-\frac{Q_{a}^{2}}{A_{a}^{2}} \sum_{i=0}^{n} L_{i j}^{\prime} A_{i}+ & \\
A_{a} g\left(\frac{d z}{d x}+S_{f}-S_{b}\right) & \approx 0 .
\end{aligned}
$$

At the selected collocation points, the approximated value is the same as the functional value,

$$
\begin{aligned}
& A_{a}\left(x=x_{i}, t\right)=\sum_{i=0}^{n} L_{i} A_{i}(t)=A_{i}\left(x=x_{i}, t\right) \quad \text { and } \\
& Q_{a}\left(x=x_{i}, t\right)=\sum_{j=0}^{n} L_{i} Q_{j}(t)=Q_{i}\left(x=x_{i}, t\right) .
\end{aligned}
$$

Therefore, the approximated Equations 18 and 19 become as follows,

$$
\begin{aligned}
\frac{d A_{i}}{d t}+\sum_{i=0}^{n} L_{i j}^{\prime} Q_{i} & =0 \text { and } \\
\frac{d Q_{i}}{d t}+\frac{2 Q_{i}}{A_{i}} \sum_{i=0}^{n} L_{i j}^{\prime} Q_{i}-\frac{Q_{i}^{2}}{A_{i}^{2}} \sum_{i=0}^{n} L_{i j}^{\prime} A_{i} & \\
+A_{i} g\left(\frac{d z}{d x}+S_{f}-S_{b}\right) & =0 .
\end{aligned}
$$

which produces a set of ODEs as shown in Equations 24 and 25 .

$$
\begin{aligned}
\dot{A_{i}}= & -\sum_{i=0}^{n} L_{i j}^{\prime} Q_{i} \\
\dot{Q}_{i}= & -\frac{2 Q_{i}}{A_{i}} \sum_{i=0}^{n} L_{i j}^{\prime} Q_{i}+\frac{Q_{i}^{2}}{A_{i}^{2}} \sum_{i=0}^{n} L_{i j}^{\prime} A_{i} \\
& -A_{i} g\left(\frac{d z}{d x}+S_{f}-S_{b}\right), \quad i=0,1, \ldots, n
\end{aligned}
$$

Two more equations can be build up using the boundary conditions, which we can choose according to the condition of the flow. For sub-critical flows, one boundary can be chosen from the upstream and the other from the downstream. For super-critical flows, both the boundaries have to be on the upstream (Georges et al., 2000).
To obtain a stable solution, the discretized time $\Delta t$, should satisfy the 'current number condition' $\mathrm{Cr}$ (Dulhoste et al., 2004),

$$
C r=\frac{\Delta t}{\Delta x} \leq \frac{1}{|v|+c},
$$

where $v$ is the velocity and $c$ is the celerity. The celerity for a trapezoidal channel is defined as $\sqrt{g \frac{A}{T}}$, where $T$ is the top width of the free surface of the channel.

\subsubsection{Selection of Collocation Points for Different Number of Points (n)}

The points are selected using the Legendre polynomials. The Legendre functions of the first kind is selected over the Chebyshev polynomials of the first kind, due to the less numerical oscillations given by the Legendre functions.

The Legendre polynomials are a set of orthogonal polynomials, which are the solutions to the Legendre differential equations (Whittaker and Watson, 1920). The Legendre polynomials are in the range of $x \in[-1,1]$ and the shifted Legendre polynomials are analogous to the Legendre polynomials, but are in the range of $x \in[0,1]$. Therefore, the shifted Legendre polynomials are selected in this study, due to the easiness in converting the collocation points over the selected channel. The shifted Legendre polynomials of the first kind can be generated from the Rodrgues' formulae (Equation 27) (Whittaker and Watson, 1920; Isaacson and Keller, 1966; Quarteroni and Valli, 2008),

$$
P_{n}(x)=\frac{1}{n !} \frac{d^{n}}{d x^{n}}\left\{\left(x^{2}-x\right)^{n}\right\} .
$$

\subsubsection{Development of the ODEs for a Sample Set of Collocation Points}

The polynomials $P_{n}(x)$ for $n$ from 3 to 5 can be derived from the Equation 27 as follows,

$$
\begin{array}{ll}
P_{1}(x)=2 x-1, & n=3, \\
P_{2}(x)=6 x^{2}-6 x+1, & n=4, \\
P_{3}(x)=20 x^{3}-30 x^{2}+12 x-1, & n=5 .
\end{array}
$$

Each collocation point $x_{i}$, lies at the roots of these polynomials along the normalized length of the channel. For a channel with a length of $l$, the positions of the collocation points can be expressed as follows,

$$
\begin{array}{rrr}
x_{i} & \in[0,0.5 l, l], & i=1,2,3 \\
x_{i} & \in[0,0.2113 l, 0.7887 l, l], & i=1,2,3,4 \\
x_{i} & \in[0,0.1127 l, 0.5 l, 0.8873 l, l] . . & i=1,2,3,4,5
\end{array}
$$

For a case of three collocation points $(n=3)$, the corresponding Lagrange interpolating polynomial coefficients, 
$L^{\prime}$, can be calculated by differentiating $L(x)$ with respect to $x$ from the Equation 5,

$L_{1}^{\prime}(x)=\frac{d}{d x}\left(\frac{x-x_{2}}{x_{1}-x_{2}} \times \frac{x-x_{3}}{x_{1}-x_{3}}\right)=\frac{\left(x-x_{3}\right)+\left(x-x_{2}\right)}{\left(x_{1}-x_{2}\right)\left(x_{1}-x_{3}\right)}$,

$L_{2}^{\prime}(x)=\frac{d}{d x}\left(\frac{x-x_{1}}{x_{2}-x_{1}} \times \frac{x-x_{3}}{x_{2}-x_{3}}\right)=\frac{\left(x-x_{3}\right)+\left(x-x_{1}\right)}{\left(x_{2}-x_{1}\right)\left(x_{2}-x_{3}\right)}$,

$L_{3}^{\prime}(x)=\frac{d}{d x}\left(\frac{x-x_{1}}{x_{3}-x_{1}} \times \frac{x-x_{2}}{x_{3}-x_{2}}\right)=\frac{\left(x-x_{2}\right)+\left(x-x_{1}\right)}{\left(x_{3}-x_{1}\right)\left(x_{3}-x_{2}\right)}$.

The coefficient matrix $L^{\prime}$ at each collocation point $x_{i}$, can be calculated by solving $L_{i}^{\prime}$ at each point $\left(L_{i}^{\prime}\left(x=x_{i}\right)\right)$, using the position values from Equation 29. The coefficient matrix for the case of the three collocation points is as follows,

$$
L^{\prime}=\left[\begin{array}{l}
L_{1} \\
L_{2} \\
L_{3}
\end{array}\right]^{T}=\frac{1}{l}\left[\begin{array}{ccc}
-3 & 4 & -1 \\
-1 & 0 & 1 \\
1 & -4 & 3
\end{array}\right]
$$

Similarly, for $n=4$,

$$
L^{\prime}=\frac{1}{l}\left[\begin{array}{cccc}
-7.0005 & 8.1964 & -2.1959 & 1 \\
-2.7326 & 1.7328 & 1.73190 & -0.7321 \\
0.7321 & -1.7319 & -1.7328 & 2.7326 \\
-1 & 2.1959 & -8.1964 & 7.0005
\end{array}\right]
$$

and for $n=5$,

$L^{\prime}=\frac{1}{l}\left[\begin{array}{ccccc}-13.0001 & 14.7884 & -2.6666 & 1.8783 & -1 \\ -5.3239 & 3.8731 & 2.0656 & -1.2910 & -0.6762 \\ 1.5 & -3.2275 & 0 & 3.2275 & -1.5 \\ -0.6762 & 1.291 & -2.0656 & -3.8731 & 5.3239 \\ 1 & -1.8783 & 2.6666 & -14.7884 & 13.0001\end{array}\right]$

The substitution of the $L^{\prime}$ in Equations 24 and 25, will give the corresponding set of ODEs. The ODEs for the case of the three collocation points are as follows,

$$
\begin{aligned}
\dot{A_{1}}= & \frac{1}{l}\left(-3 Q_{1}+4 Q_{2}-Q_{3}\right), \\
\dot{A_{2}=} & \frac{1}{l}\left(-Q_{1}+Q_{3}\right), \\
\dot{A_{3}=} & \frac{1}{l}\left(Q_{1}-4 Q_{2}+3 Q_{3}\right), \\
\dot{Q_{1}}= & -\frac{2 Q_{1}}{A_{1} l}\left(-3 Q_{1}+4 Q_{2}-Q_{3}\right)+\frac{Q_{1}^{2}}{A_{1}^{2} l}\left(-3 A_{1}+4 A_{2}-A_{3}\right) \\
& -A_{1} g\left(\frac{d z}{d x}+S_{f_{1}}-S_{b}\right), \\
\dot{Q_{2}=} & -\frac{2 Q_{2}}{A_{2} l}\left(-Q_{1}+Q_{3}\right)+\frac{Q_{2}^{2}}{A_{2}^{2} l}\left(-A_{1}+A_{3}\right) \\
& -A_{2} g\left(\frac{d z}{d x}+S_{f_{2}}-S_{b}\right), \\
& -\frac{2 Q_{3}}{A_{3} l}\left(Q_{1}-4 Q_{2}+3 Q_{3}\right)+\frac{Q_{3}^{2}}{A_{3}^{2} l}\left(A_{1}-4 A_{2}+3 A_{3}\right) \\
& -A_{3} g\left(\frac{d z}{d x}+S_{f_{3}}-S_{b}\right) .
\end{aligned}
$$

One or two equations from the above set of equations, can be replaced by the chosen boundary conditions.

\subsection{The Kurganov and Petrova (KP) Scheme}

The KP scheme (Kurganov and Petrova, 2007) is a well balanced scheme which utilizes a central upwind scheme. Further, it does not have the Reimann problem. To illustrate this scheme, the SVEs stated in Equations 1 and 2 are re-written as follows,

$$
\frac{\partial U}{\partial t}+\frac{\partial F}{\partial x}=S
$$

where,

$$
\begin{aligned}
U & =\left[\begin{array}{c}
A \\
Q
\end{array}\right], \\
F & =\left[\begin{array}{c}
Q \\
\frac{Q^{2}}{A}
\end{array}\right], \quad \text { and } \\
S & =\left[\begin{array}{c}
0 \\
-A g\left(\frac{\partial z}{\partial x}+S_{f}-S_{b}\right)
\end{array}\right] .
\end{aligned}
$$

The space is discretized in to a grid for a finite volume cell of a cell size of $\Delta x$ and $x_{j-\frac{1}{2}} \leq x_{j} \leq x_{j+\frac{1}{2}}$ in a uniform grid. The KP scheme for the given Equation 36, can be written as the following set of ODEs,

$$
\frac{d \bar{U}_{j}(t)}{d t}=-\frac{H_{j+\frac{1}{2}}(t)-H_{j-\frac{1}{2}}(t)}{\Delta x}+\bar{S}_{j}(t),
$$

where $H_{j \pm \frac{1}{2}}(t)$ are the central upwind numerical fluxes at the cell interfaces (Kurganov and Petrova, 2007; Sharma, 2015; Vytvytskyi et al., 2015). More details in this scheme is included in (Kurganov and Petrova, 2007). The time step $\Delta t$ is restricted by the standard Courant-FriederichLevy (CFL) condition as follows (Kurganov and Petrova, 2007; Bollermann et al., 2013),

$$
\mathrm{CFL}=\frac{\Delta t}{\Delta x} \max _{j}\left|a_{j+\frac{1}{2}}^{ \pm}\right| \leq \frac{1}{2},
$$

where $a_{j \pm \frac{1}{2}}^{ \pm}$is a one sided local speed of propagation.

\subsection{The Parameters of the Open Channel}

The selected open channel is a prismatic channel with a trapezoidal cross section. The total length $l$ of the channel is $2.95 \mathrm{~m}$. The bottom width of the channel is $0.2 \mathrm{~m}$, with a zero channel bed slope $S_{b}$. The Strickler friction coefficient, $k_{S}$ is taken as $42 \frac{\mathrm{m}^{1 / 3}}{\mathrm{~s}}$.

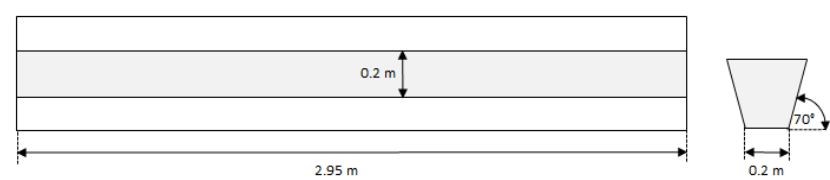

Figure 1. Plan View and the Side Elevation of the Prismatic Channel 


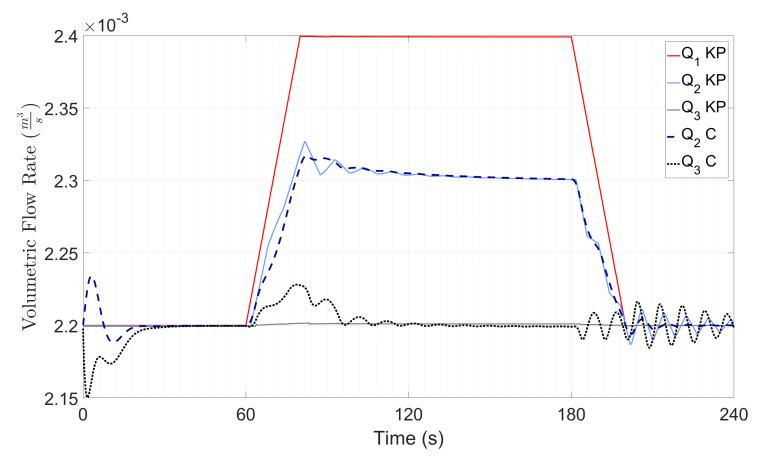

Figure 2. Comparison of the Flow Rates between the KP method and OC Method, at the Three Collocation Points. 'KP': Results from KP, 'C ': Results from OC

\section{Simulation, Results and Discussion}

A prismatic channel is selected for the dynamic simulations in MATLAB(9.0.1), with three cases of different number of collocation points. For the collocation method, the selected boundary conditions are the flow rate into the channel and the wetted cross sectional area out of the channel. For the simulations with KP, the two boundaries are the flow rates into and out of the channel. For both the methods, the sets of ODEs are solved by the use of Runge Kutta fourth order numerical scheme with a fixed step length.

\subsection{Simulation Setup}

The simulations for the KP method were started from a steady state, and after 60 seconds, the volumetric flow rate at the inlet was changed from 0.0022 to $0.0024 \frac{\mathrm{m}^{3}}{\mathrm{~s}}$ within 20 seconds. This increased flow rate was maintained for about 120 seconds, and then it was reduced back to the previous value within 20 seconds. The flow rate at the end of the channel was kept at the same value of $0.0022 \frac{\mathrm{m}^{3}}{\mathrm{~s}}$, throughout the simulations.

The inlet flow rate conditions of the KP method and the outlet wetted cross section area resulted from the simulations, were used as the boundary conditions for the simulations of the collocation method.

\subsection{Results and Discussion}

Three case studies were simulated using the orthogonal collocation (OC) method. Those results are compared with the results from the KP method and are described in the sections 3.2.1, 3.2.2 and 3.2.3.

\subsubsection{Case 1: Three Collocation Points $(n=3)$}

The results from the simulations of the KP scheme are compared with the results from the method with three collocation points. The volumetric flow rates and the heights of the fluid level at the three points are shown in Figures 2 and 3 , respectively.

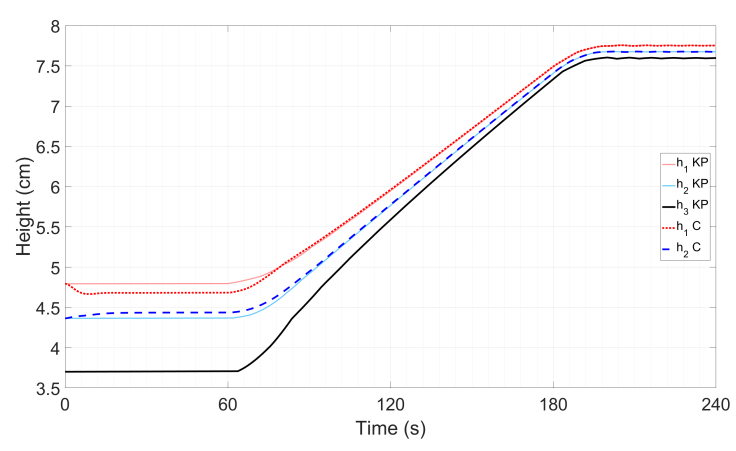

Figure 3. Comparison of the Fluid Levels between the KP method and the OC Method, at the Three Collocation Points. 'KP ': Results from KP, 'C ': Results from OC

The flow rates obtained from the collocation method are similar to the results from the KP method, but with a few numerical oscillations. At the start of the simulation, the numerical oscillations can be observed due to the unsteady state conditions in the collocation method. These deviations can also be clearly seen in the deviations of the heights in Figure 3 at the beginning. During the transient conditions, the flow rate at the middle of the channel, which is obtained by the collocation method, i.e. $Q_{2} C$ in Figure 3 after 60 seconds, has less numerical

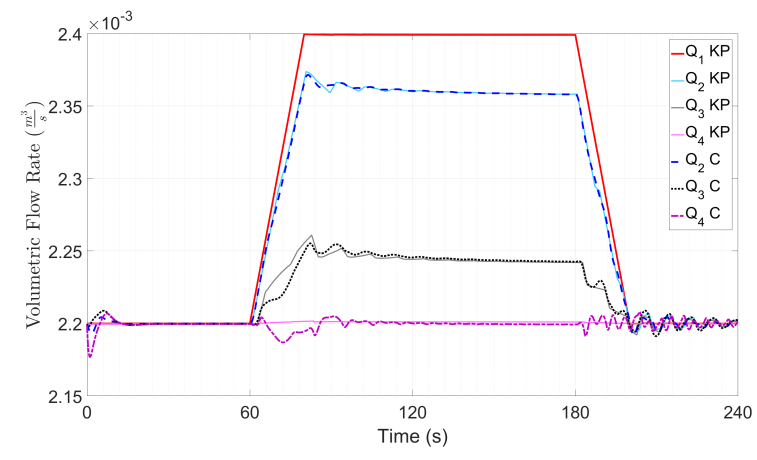

Figure 4. Comparison of the Flow Rates between the KP method and the OC Method, at the Four Collocation Points. 'KP ': Results from KP, 'C ': Results from OC

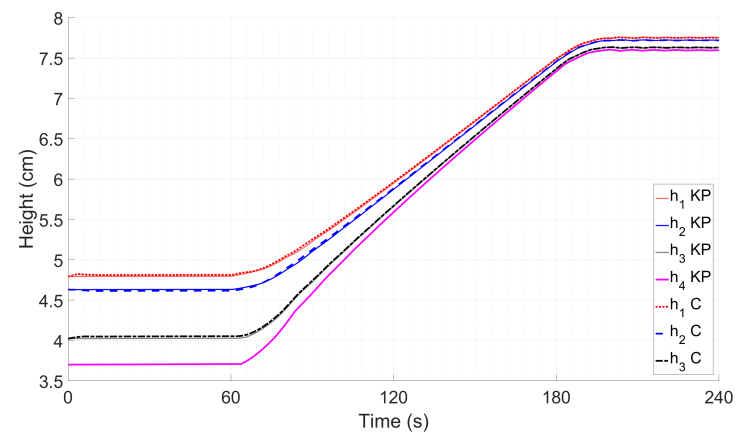

Figure 5. Comparison of the Fluid Levels between the KP method and the OC Method, at the Four Collocation Points. 'KP ': Results from KP, 'C ': Results from OC 
oscillations than the same from the KP method, but the flow rate at the end of the channel i.e. $Q_{3} C$ has more oscillations than from the KP method.

\subsubsection{Case 2: Four Collocation Points $(n=4)$}

The volumetric flow rates and the heights of the fluid level at the selected four points, are shown in Figures 4 and 5, respectively.

The results of the simulation from the OC method with four collocation points are more comparable with the results from the KP method, than the same with the three collocation points. Although the amplitude of the oscillations are reduced, the frequency of the oscillations are increased than in the previous case (in section 3.2.1). The reason could be the dual effect of the better approximation due to the increase of the number of collocation points, and the oscillatory behavior of the polynomial approximation due to the increase of the order of the polynomial. This could be observed further by increasing the number of collocation points to five.

\subsubsection{Case 3: Five Collocation Points $(n=5)$}

The results for the five collocation points are shown in Figures 6 and 7, respectively. The better approximation due to the increase of the number of collocation points has dominated over the oscillatory behavior caused by the increase of the order of the polynomial, as shown in Figure 6. The oscillations in OC method are the same as from KP, except for $Q_{5} C$, which is at the end of the channel.

\subsubsection{Selection of an Orthogonal Polynomial for the Collocation Points}

A comparison between the Legendre and Chebyshev polynomials of the first kind was done to justify the selection of the Legendre polynomial. The simulations were done for the case of five collocation points. As shown in the zoomed areas of the Figure 8, it can be justified that the Legendre polynomials tend to produce less oscillations compared to the Chebyshev polynomials.

The OC method is accurate enough with four or more collocation points, as oppose to the numerous discretization points (100) in the KP method. Therefore, to satisfy the CFL condition, the time step $\Delta t$ of the KP scheme has to be small due to the small $\Delta x$. On the contrary, to satisfy the different Current number condition, the OC method allows a larger time step due to the comparatively bigger $\Delta x$. Altogether, the computational time taken for the OC method was about 5-20 times less than the computational time taken by the KP method. Handling the ODEs that are

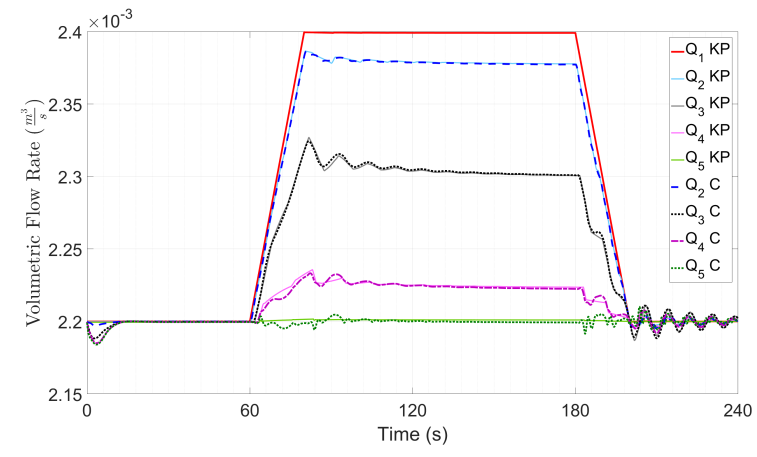

Figure 6. Comparison of the Flow Rates between the KP method and the OC Method, at the Five Collocation Points. 'KP ': Results from KP, 'C ': Results from OC

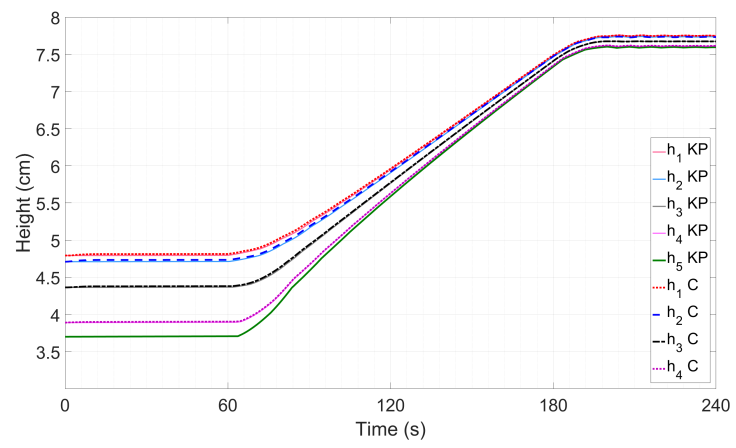

Figure 7. Comparison of the Fluid Levels between the KP method and the OC Method, at the Five Collocation Points. 'KP ': Results from $\mathrm{KP},{ }^{\prime} \mathrm{C}$ ': Results from OC

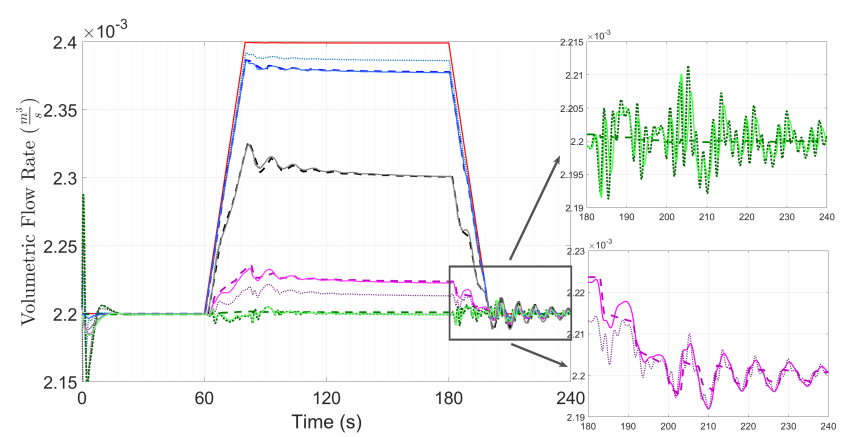

Figure 8. Comparison of the Legendre and Chebyshev polynomials of the first kind. (dashed lines: Results from KP at different collocation points, dotted lines: Results from the OC using Chebyshev polynomials, solid lines: Results from OC using Legendre polynomials.

generated by the OC method is computationally simpler than the KP method. Further, it has a considerably similar accuracy, specially takes much less computational time, which makes the use of OC method in the application of online state and parameter estimation, to be promising.

\section{Conclusion}

The possibility of efficiently reducing the PDEs into ordinary differential equations (ODEs) using orthogonal col- 
location method, is studied with the goal of application in state and parameter estimations in real-time. The collocation method showed the flexibility of choosing the boundary conditions with respect to the flow behavior. The results were comparable enough to the selected finite volume method, which is a widely used, central-upwind scheme. Further, a significant reduction in the computational time in the collocation method is observed. Therefore, the collocation method shows a promising potential of using it in the estimation of state and parameters of open channel flows.

\section{Acknowledgment}

The economic support from The Research Council of Norway and Statoil ASA through project no. 255348/E30 'Sensors and models for improved kick/loss detection in drilling (Semi-kidd)' is gratefully acknowledged.

\section{References}

Andreas Bollermann, Guoxian Chen, Alexander Kurganov, and Sebastian Noelle. A Well-Balanced Reconstruction of Wet/Dry Fronts for the Shallow Water Equations. Journal of Scientific Computing, 56(2):267-290, 2013. doi:10.1007/s10915-012-9677-5.

O. V. Bulatov. Analytical and Numerical Riemann Solutions of the Saint Venant Equations for Forward- and Backward-Facing Step Flows. Computational Mathematics and Mathematical Physics, 54(1):158-171, 2014. doi:10.1134/S0965542514010047.

Mieczyslaw Chalfen and Andrzej Niemiec. Analytical and Numerical Solution of Saint-Venant Equations. Journal of Hydrology, 86:1-13, 1986.

M. Hanif Chaudhry. Open-Channel Flow. Springer, New York, 2nd edition, 2008. ISBN 9780387301747.

V. T. Chow. Open-Channel Hydraulics. McGraw-Hill, New York, 1959. ISBN 0070107769.

W. H. Chung and Y. L. Kang. Analytical Solutions of Saint Venant Equations Decomposed in Frequency Domain. Journal of Mechanics, 20(03):187-197, 2004. doi:10.1017/S1727719100003403.

Jean-François Dulhoste, Didier Georges, and Gildas Besançon. Nonlinear Control of Open-Channel Water Flow Based on Collocation Control Model. Journal of Hydraulic Engineering, 130(3):254-266, 2004. doi:10.1061/(ASCE)07339429(2004)130:3(254).

Didier Georges, Jean-françois Dulhoste, and Gildas Besançon. Modelling and Control of Water Flow Dynamics via a Collocation Method. Math. Theory of Networks and Systems, 2000.

Eugene Isaacson and Herbert Bishop Keller. Analysis of Numerical Methods. John Wiley \& Sons, New York, 2nd edition, 1966. ISBN 9780486680293.
Alexander Kurganov and Guergana Petrova. A SecondOrder Well-Balanced Positivity Preserving Central-Upwind Scheme for the Saint-Venant System. Communications in Mathematical Sciences, 5(1):133-160, 2007. doi:10.4310/CMS.2007.v5.n1.a6.

Xavier Litrico and Vincent Fromion. Modeling and Control of Hydrosystems. Springer, 2009. ISBN 9781848826236.

Alfino Quarteroni and Alberto Valli. Numerical Approximation of Partial Differential Equations. Springer, Berlin, 1st edition, 2008. ISBN 9783540852674.

Roshan Sharma. Second order scheme for open channel flow. Technical report, Telemark University College, 2015. URL http: //hdl.handle.net/11250/2438453.

Gabor Szegö. Orthogonal Polynomials, volume 23. American mathematical society, 4th edition, 1939. ISBN 9780821810231 .

Liubomyr Vytvytskyi, Roshan Sharma, and Bernt Lie. Model Based Control for Run-of-river System. Part 1: Model Implementation and Tuning. Modeling, Identification and Control, 36(4):237-249, 2015. doi:10.4173/mic.2015.4.4.

E.T. Whittaker and G.N. Watson. Legendre Functions. In A Course of Modern Analysis, chapter XV, pages 302-336. Cambridge University Press, 3rd edition, 1920. 